\title{
Hidden symmetries for thermodynamics and emergence of relativity
}

\author{
Liu Zhao \\ School of Physics, Nankai University, \\ Tianjin 300071, P R China \\ email: lzhao@nankai.edu.cn
}

(Dated: November 1, 2018)

\begin{abstract}
Erik Verlinde recently proposed an idea about the thermodynamic origin of gravity. Though this is a beautiful idea which may resolve many long standing problems in the theories of gravity, it also raises many other problems. In this article I will comment on some of the problems of Verlinde's proposal with special emphasis on the thermodynamical origin of the principle of relativity. It is found that there is a large group of hidden symmetries of thermodynamics which contains the Poincare group of the spacetime for which space is emergent. This explains the thermodynamic origin of the principle of relativity.
\end{abstract}

\section{INTRODUCTION}

Of all physical theories about Nature, only two branches are independent of concrete details of the systems being considered, i.e. thermodynamics and relativity (in either the special or general sense). These two theories are about the universal principles every physical system must obey and are hence referred to as principle theories. All other theories are about concrete systems. It is puzzling why in the first place Nature prefers to abide by two principle theories rather than simply one (puzzle of two), because two is more dangerous in the sense that potential contradictions between them might occur. However, Nature is quite smart in avoiding such contradictions. Even more, in many cases the two principle theories seem to be mysteriously connected to each other. This connection is especially transparent when one talks about relativity in the general sense: on the one hand, for every black hole solution of the general theory of relativity one can define thermodynamic quantities like entropy and temperature and check that the classical laws of thermodynamics are obeyed; on the other hand, it has repeatedly been discussed that the Einstein equation [1] [2] [3] and the action [4] of the general theory of relativity can be derived from the first law of thermodynamics, and recently Verlinde [5] went further by proposing that gravity is an entropic force and thus calling for an end of gravity as a fundamental force. Subsequent surge of works appeared almost instantly, including [6], which applied Verlinde's idea in loop quantum gravity, [7] and [8], both derived the Friedman equation using Verlinde's idea, [9], which applied Verlinde's idea in establishing UV/IR relation and obtaining holographic dark energy, [10], which tries to interpret electrostatic force also as an entropic force, [11], [12] and [13], which applied Verlinde's ideas in the settings of modified gravity, brane cosmology and Horava gravity respectively, and [14], which proposed two novel kind of cosmic perturbations corresponding to Verlinde's emergent gravity. The idea of emergent gravity also attracted some quantum information people, see [15] for an alternative proposal.

Verlinde's proposal is certainly very beautiful. If proven true, a significant portion of modern theories of fundamental physics should be reformulated. It not only has the potential of addressing the long standing problem of quantum gravity by identifying gravity as a purely macroscopic effect, but also implies that space itself must be emergent as a macroscopic effect. Moreover, it has the potential to resolve the puzzle of two mentioned above. However, Verlinde's idea also raises many new problems because most concepts taking space as a fundamental existence must be reexamined. Verlinde himself has noticed this point and explained in particular the concepts of inertia and Newton's law of classical mechanics from the new point of view.

In this article I will comment that there are more problems which should be checked against before one can take Verlinde's proposal more seriously. The origin of the principle of relativity is among these problems. It is found that there is a large group of hidden symmetries for thermodynamics which has not been discussed before. By incorporating this hidden symmetry group together with Verlinde's idea and the second law of thermodynamics, it is shown that the principle of relativity arises naturally. Meanwhile, a clearer understanding about the role of holographic principle in Verlinde's proposal is given. It turns out that holography is a requirement of democracy between different scaling dimensions and the fact that space is three dimensional.

\section{VERLINDE'S PROPOSAL: PROBLEMS TO BE ANSWERED}

Verlinde's proposal contains the following key ingredients:

- space is emergent, the part of space which has not yet emerged is enclosed by a holographic screen and the entropy is proportional to the area of the screen;

- gravity is an entropic force just like any other generalized forces entering in the first law of thermodynamics. More concretely, gravity is caused by the change of entropy behind the holographic screen due to the emergence of space; 
- the temperature is either related to the acceleration of the observer via Unruh's law or related to the total energy of the system via equipartition law (the equipartition of energy on black hole horizons is discussed earlier by Padmanabhan in [16], see also $[2])$, and the total energy of the system is also equal to the mass behind the holographic screen times $c^{2}$, i.e. $E=M c^{2}$. Using Unruh's law for temperature leads to Newton's law of classical mechanics, and using equipartition law and $E=M c^{2}$ gives rise to Newton's law of gravitational force.

A lot more have been discussed in Verlinde's paper [5], however besides the above new ingredients the rest arguments and sketchy derivations seem to have been discussed in or implied by earlier works since Jacobson's [1], in particular, the formal derivation of Einstein equation is now new.

Now let us take a more careful look at the new ideas listed above.

First of all, given that space is emergent, one has to explain why is it look like what we perceive it. In particular, why is space three dimensional, or even why the dimension of space is an integer? This is a problem one has to answer in an emergent theory of space but these were not paid for a single word in Verlinde's paper;

Second, it is known since the 1930's [17] that classical thermodynamics is incompatible with the special theory of relativity if spacetime is considered as a fundamental existence. However, in an emergent theory of space one has to see the principle of relativity also as an emergent consequence. It remains to check whether the emergence of the principle of relativity is possible or not following Verlinde's proposal, and I think this is by now one of the most severe obstacle to be overcome before this new proposal can be more widely accepted in the physics community;

Third, in deriving the Newton's law of gravity, the equipartition law together with the relation $E=M c^{2}$ were used as input. But the origin of the latter relation is only known from special relativity. It gives an impression that special relativity is provided as another fundamental assumption, but even though it is still strange that in order to obtain the nonrelativistic law for gravity one has to use the relativistic relation for the total energy. Also the Unruh temperature relation is a consequence of relativistic motion, while it is used in deriving the nonrelativistic law of classical mechanics. Moreover, the use of equipartition law also feels strange, because equipartition law depends on the microscopic details of the system while Newton's law doesn't.

There are other issues which need more interpretations but I prefer not to concentrate on them and just emphasis on the problem of emergence of the principle of relativity. If the principle of relativity is put in by hand, it will seriously hurt the picture of the emergence of space, and the puzzle of two will not be resolved. On the contrary, if the principle of relativity is emergent, then not only the puzzle of two is resolved, it will also call for an improve- ment for the derivation of the Newton's law of gravity, or simply view Newton's law as the nonrelativistic limit of general relativity as usual while considering general relativity as an emergent theory about gravity. In either way the puzzle of deriving nonrelativistic laws using relativistic formulas without taking a nonrelativistic limit should be avoided.

In the next section I will propose a possible solution to the emergence of the principle of relativity by unraveling a large group of hidden symmetries of classical thermodynamics. Assuming that space is emergent and plays as extensive variables in the first law of thermodynamics, it will be seen that the Poincare group is naturally contained in the hidden symmetry group of thermodynamics, which gives an explanation of the principle of relativity as an emergent concept from thermodynamic geometry.

\section{GEOMETRY AND HIDDEN SYMMETRIES OF CLASSICAL THERMODYNAMICS}

There are quite a few different prescriptions of thermodynamics as a geometric system. Gibbs [18] described the thermodynamic phase space as a contact manifold. Weinhold [19] and Ruppeiner [20] respectively described the geometry of the thermodynamic configuration space (i.e. the space of equilibrium states) as Riemannian geometries (with different metric choices). And recently $\mathrm{H}$. Quevedo [21] [22] described the geometry of the thermodynamic phase space as a contact Riemannian geometry invariant under the group of Legendre transformations.

For my purpose it is tempting to describe the geometry of the thermodynamic phase space as a contact, Riemannian geometry whose pull back to the thermodynamic configuration space has a metric with Lorentzian signature.

To begin with, let me describe the contact geometry of the thermodynamic phase space $\mathcal{T}$, following the spirits of Gibbs [18]. The space $\mathcal{T}$ is a $2 n+1$ dimensional contact manifold with coordinates $\left(\Phi, E^{a}, I_{a}\right)(a=1,2, \ldots, n)$, where $\Phi$ is a thermodynamic potential, $E^{a}$ and $I_{a}$ are respectively extensive and intensive variables of the system, and a contact one form $\Theta=d \Phi-\sum_{a} I_{a} d E^{a}$ obeying $(d \Theta)^{\wedge n} \wedge \Theta \neq 0$ is needed in order to identify the contact structure of the manifold $\mathcal{T}$. The space of classical equilibrium thermodynamical states $\mathcal{E}=\operatorname{span}$ of $\left\{E^{a}\right\}$ is embedded in $\mathcal{T}$ as a subspace by a smooth mapping

$$
\varphi: \mathcal{E} \rightarrow \mathcal{T}, \quad \varphi\left(E^{a}\right)=\left(\Phi\left(E_{a}\right), E^{a}, I_{a}\left(E^{a}\right)\right),
$$

and the pull back condition $\varphi^{*}(\Theta)=0$ gives rise to the first law of thermodynamics, i.e.

$$
d \Phi=\sum_{a} I_{a} d E^{a},
$$

together with the equations of states

$$
I_{a}=\frac{\partial \Phi}{\partial E^{a}} .
$$


It is known from standard texts on differential geometry that a contact manifold possesses a very special group of symmetries, i.e. the group of contact transformations. The group of Legendre transformations, i.e.

$$
\Phi=\tilde{\Phi}-\sum_{a \in \mathcal{I}^{\prime}} \tilde{I}_{a} \tilde{E}^{a}, \quad E^{a}=-\tilde{I}_{a}, \quad I_{a}=\tilde{E}^{a},
$$

for $a \in \mathcal{I}^{\prime}$ with $\mathcal{I}=\{0,1,2, \ldots, n-1\}$ and $\mathcal{I}^{\prime} \subset \mathcal{I}$ is any subset therein, is a subset of the group of contact transformations, and H.Quevedo stressed very much on the invariance under this subgroup in his prescription of geometrothermodynamics [21] [22]. It is however not my intention to keep my eyes addicted to the group of Legendre transformations. Rather, the introduction of a Riemannian metric on $\mathcal{T}$ seems more attractive. The concrete form of the metric on $\mathcal{T}$ is not important. What is important is that the pull back of this metric naturally introduces a metric on the thermodynamic configuration space $\mathcal{E}$, which, in its most general form, can be written as

$$
d s^{2}=g_{a b}(E) d E^{a} d E^{b} .
$$

Now one of the crucial part of this work turns up. There exists a large continuous group of hidden symmetries which keeps both (1) and (3) invariant. This hidden symmetry group is the group $G$ of general coordinate transformations in the space $\mathcal{E}$, i.e.

$$
\begin{gathered}
E^{a} \rightarrow E^{\prime a}=E^{\prime a}\left(E^{b}\right), \quad \Phi \rightarrow \Phi^{\prime}=\Phi, \\
d E^{a} \rightarrow\left(\frac{\partial E^{a}}{\partial E^{\prime b}}\right) d E^{\prime b}, \quad I_{a} \rightarrow\left(\frac{\partial E^{\prime b}}{\partial E^{a}}\right) I_{b}^{\prime} .
\end{gathered}
$$

In the above, the transformation law of $I_{a}$ is triggered by the relation (2).

Borrowing some terminologies from the general theory of relativity, $d E^{a}$ transform under the group $G$ as a contravariant vector, $I_{a}$ transform as a covariant vector, while $\Phi$ transforms as a scalar.

Notice that although in the above I am using geometric terminologies like manifold, Riemannian metric, contravariant and covariant vectors etc, no fundamental existence of space is assumed actually. As Verlinde has emphasized, the definition of thermodynamics does not need the existence of space. Space actually emerges as macroscopic consequences as some of the extensive variables (generalized displacements) in the space $\mathcal{E}$. Therefore, the group $G$ is by now purely thermodynamic in nature, nothing relevant to spacetime symmetry has entered into play.

The invariance under the group $G$ is totally a new observation which has not been discussed before. Considering the fact that there has already been quite some works [19] [20] assigning a Riemannian metric to the space of equilibrium states of thermodynamics, it is quite strange why this large group of isometries have not been discussed before in the context of thermodynamic geometries (for the case of Weinhold geometry, the isometry group is discussed in [23], but the groups considered there is not the same as the one discussed here. Meanwhile, the deep implications implied by the symmetries is somehow not widely acknowledged). Anyway, the finding of this large group of hidden symmetries provides room for an interpretation of the principle of relativity as an emergent effect from thermodynamics, as will be seen below.

First let me fix the choice of the thermodynamic potential $\Phi$ by identifying it as the total internal energy of the system. This breaks the Legendre symmetry but leaves the $G$ invariance unaffected. Meanwhile, this specific choice of thermodynamic potential implies that the entropy $S$ is among the extensive variables, let me give it the index 0, i.e. $E^{0}=S$. Unlike the case of Weinhold and Ruppeiner, I did not associate the metric $g_{a b}(E)$ with the hessian of any specific thermodynamic function, so there is still enough freedom in choosing the signature of $g_{a b}(E)$. Let the signature be Lorentzian and let $E^{0}$ be the coordinate bearing the different signature from others. The $G$ symmetry allows to make different choices for the reference frames on $\mathcal{E}$. Near any point $X$ in $\mathcal{E}$, take a small neighborhood $U$ thereof, then using $G$ transformations one can always fix the metric (3) on $U$ to a special form, i.e.

$$
d s^{2}=\eta_{a b} d E^{a} d E^{b},
$$

where $\eta_{a b}$ is the Lorentzian metric in $n$ dimensions. This is the analogue of taking a local inertial frame in the general theory of relativity, but now practiced purely in the framework of thermodynamics. Once the frame (4) is taken, the $G$ symmetry is broken, but a residual subgroup $H$ survives, which consists of linear transformations among $E^{a}$, i.e.

$$
E^{a} \rightarrow E^{\prime a}=\Lambda^{a}{ }_{b} E^{b}+T^{a},
$$

in which $\Lambda^{a}{ }_{b}$ belongs to the orthogonal group $S O(n-1,1)$ and $T^{a}$ are constants. Clearly eq.(1) is invariant under such a group of transformations, provided $I_{a}$ transform inversely under $H$.

Readers may have already noticed the close analogue of eq.(5) with the Poincare group. To actually establish the relationship between the group $H$ and the Poincare group, extra input is needed, including the second law of thermodynamics and Verlinde's proposal for an emergent space.

Consider first the second law of thermodynamics. There are many presentations for the second law in the literature. For convenience, we take the following presentation:

Second law: In any thermodynamic process connecting two equilibrium states of an isolated macroscopic system, the entropy does not decrease, i.e.

$$
d S \geq 0 .
$$

This presentation can also be put in another way, i.e. any equilibrium state of a given macroscopic system of lower entropy cannot be the consequence of a thermodynamic 
process of another equilibrium state of higher entropy. Such a statement is reminiscent to the causality principle of relativistic physics and may be called the thermal causality principle.

Careful readers may have felt uneasy with the last paragraph. According to eq.(5), $S$ is not a scalar under the action of the group $H$. In other words, there is not a unique choice for the extensive variable $S$ in the presence of the symmetry group $H$. So the thermal causality principle must be formulated in an invariant way under the action of the group $H$. For this purpose we need an invariant quantity under the action of $H$, and the line element on the space $\mathcal{E}$ happens to fill this gap.

Geometrically the line element (4) describes the invariant distance between two points in $U$. What is the thermodynamic meaning of such a distance? It is clear that the two points connected by $d s^{2}$ correspond to two distinct equilibrium states, so the line element between them must corresponds to a thermodynamic process evolving from one state to the other. Now the following crucial question arises: given any two states $A, B$ in $U$, does the thermodynamic process connecting them always exist? To answer this question, let me first fix a reference frame on $U$ such that $S_{A} \leq S_{B}$. Then the second law implies that there can possibly be a thermodynamic process evolving from the state $A$ to the state $B$. However, such a process is not guaranteed to exist, since a change of frame on $U$ can spoil the inequality $S_{A} \leq S_{B}$. So, if $A$ and $B$ are such that $S_{A}<S_{B}$ in one frame but $S_{A}>S_{B}$ in some other frame, the presumed thermodynamic process evolving from $A$ to $B$ (or vise versa) should be excluded by the second law. In other words, such states $A$ and $B$ must not be causally connected via thermodynamic process. On the other hand, it is possible that for properly chosen states $A$ and $B$, the inequality $S_{A} \leq S_{B}$ holds for all allowed choices of frames on $U$. In such cases, one cannot exclude the possibility that $B$ is the thermodynamic consequence of $A$ through some thermal process. It is not a hard practice to show that $S_{A} \leq S_{B}$ holds for all allowed choices of frames on $U$ if and only if $S_{A} \leq S_{B}$ holds in one frame on $U$ and the line element $d s_{A B}^{2}$ between $A, B$ obeys $d s_{A B}^{2} \geq 0$. So, the thermal causality principle can be formulated as follows:

Invariant presentation of the second law: In any thermodynamic process connecting two equilibrium states of an isolated system, the line element $d s^{2}$ must be nonnegative.

For this reason we can possibly call $d s$ the proper entropy change between the two states. Notice that I did not assign an arrow to the line element, so a thermodynamic process corresponds to both $d s_{A B} \geq 0$ and $d s_{A B} \leq 0$.

Now let me follow Verlinde's idea and consider the whole universe as an emergent macroscopic system. This implies, among other things, that the spacial coordinates $X^{i}$ are among the extensive variables $E^{a}$ in the space $\mathcal{E}$. Moreover, since nothing is assumed to exist outside the universe, one can consider the universe as an iso- lated system, i.e. the condition for the thermal causality principle hold, any physical thermodynamic process must obey $d s^{2} \geq 0$.

Unlike Verlinde's original proposal for a single emergent dimension, I postulate here that all the spacial dimensions are emergent. With the emergent spacial coordinates as extensive variables, the first law (1) should be modified as follows:

$$
d E=T d S-F_{i} d X^{i}-p d V+\mu d N
$$

where the sum over $i$ extends through all spacial dimensions. Note that $F_{i}$ are emergent forces just like $p$ does, and $X^{i}$ and $V$ are all macroscopic quantities which need not have an microscopic origin. This is what the term emergence of space means.

Since the spacial coordinates are identified as generalized displacements, the total number of such displacements must be an integer. This explains why the dimension of space is an integer. It is natural to assign a scaling dimension for each of the extensive variables. Doing so one sees that only when space is three dimensional and $S$ is proportional to the area of a holographic screen, the right hand side of eq.(6) can be democratic (i.e. evenly distributed) between quantities of different scaling dimensions. In the above, $N$, the number of total microscopic degrees of freedom, is zero dimensional, $X^{i}$ are one dimensional, $S$ is two dimensional and $V$ is three dimensional. In this way the role of holographic principle in Verlinde's proposal and the implicit assumption of 3 spacial dimensions are replaced by a single requirement of democracy between scaling dimensions.

In a process with $d V=d N=0$, the action of the subgroup $P$ of $H$ leaving $V$ and $N$ invariant is isomorphic to the Poincare group in the subspace $\mathcal{M}$ of $\mathcal{E}$ spanned by $S$ and $X^{i}$. If I assume that $d S$ is proportional to the time elapsed between the two equilibrium states (and this has to be so, because in any reference frame, $S$ is a monotonic function of time $t$, thus $d S \propto d t$ locally, and the procedure of fixing the metric (4) could fix the constant of proportionality to 1 ), then the relativistic causality principle naturally follow from the thermal causality principle.

What is the role of $H$ in the process involving non zero $d V$ or $d N$ ? The answer could also be very interesting. For instance, if a process involves non zero $d V$, then the group $H$ is bigger than $P$, i.e. the pull back line element $d s^{2}$ possesses a bigger isometry group. Such a group provides room for cosmological expansion, and the corresponding intensive variable $p$ could possibly play the role of dark energy. However, at present, such possibilities must be regarded as speculative, because much more work on the understanding of thermodynamic emergence of space has to be done before such speculations can be made more sounded. As for $d N \neq 0$, the process will involve production or destruction of particles and that is beyond the present discussion on the emergence of the principle of relativity. 


\section{DISCUSSIONS}

Using the hidden symmetry group of thermodynamics and the thermal causality principle it is found that the principle of relativity arises naturally as an emergent consequence of thermodynamics. Thermal causality is identified with the relativistic causality by requiring that the increase in entropy, $d S$, is proportional to the time elapse. This analysis resolves a number of problems left over from Verlinde's proposal for an emergent space and gravity.

One may wonder why in the first place the principle of relativity could arise as an emergent consequence of thermodynamics which has long been known to be incompatible with special relativity regarded as a fundamental principle theory. In particular, why is the total energy $E$ taken as the thermodynamic potential transform as a scalar under the emergent Poincare group, while in traditional special relativity, energy transforms as the zeroth component $p^{0}$ of the energy-momentum 4 -vector. The answer is that $p^{0}$ and $E$ are completely different objects which should be not confused with each other. Actually, $p^{0}$ refers to the energy of certain microscopic degree of freedom, while $E$ counts the total energy of all microscopic degrees of freedom in the system, so by definition $E$ is an integral quantity in which the covariant and contravariant actions of Poincare group cancel completely. Due to the same reason, there is no reason to write $E=M c^{2}$, because $M c^{2}$ only represents the energy of a microscopic degree of freedom of mass $M$ at rest, while $E$ counts the energy of all microscopic degrees of freedom, and most of these are not at rest.

Another reason supporting the view point of not regarding $E$ as a component of relativistic 4-vector comes from the new relationships between relativity and thermodynamics. If spacetime were fundamental and $E$ is the total energy of a thermodynamic system moving in space, then it seems inevitable that $E$ should change under rel- ativistic change of coordinates. However, in the present picture, space itself is emergent from thermodynamics. The thermodynamic system no longer moves in space, and $E$ contains not only the energy of ordinary matter but also the energy of space. (The picture of space as emergent from thermodynamics implies that space itself can be heated, and thus stores energy. On this point, I am grateful to T. Padmanabhan for bringing my attention to the paper [24] after the first versions of the present paper have appeared on arXiv.) We human beings have no previous knowledge at all on how the total energy of matter and space changes under coordinate changes in space and time.

In this article, only the linear subgroup of the hidden symmetries of thermodynamics is analyzed in detail. A more thorough analysis on the complete hidden symmetry group of thermodynamics should be considered later, which is expected to reveal how general relativity arises from thermodynamics. Whatever the details will be, I expect that a better derivation of Einstein equation should follow and Newton's law of gravity should arise as nonrelativistic limit of general relativity as usual, thus removing the puzzle of deriving nonrelativistic laws of gravity from relativistic formulas.

The argument made in this article is very preliminary. Much more works are yet to be done. Among other things the hidden symmetry group $G$ of thermodynamics is bigger than the general coordinate transformations for general relativity provided in the first law of thermodynamics $d V$ and $d N$ are not simultaneously zero. The role of the extra symmetries is yet to be understood, and perhaps this will give novel insights into the general theory of relativity.

\section{ACKNOWLEDGMENT}

This work is supported by the National Natural Science Foundation of China (NSFC) through grant No.10875059.
[1] T. Jacobson, "Thermodynamics of Spacetime: The Einstein Equation of State," Phys.Rev.Lett. 75 (1995) 12601263 [arXiv gr-qc (Apr, 1995) gr-qc/9504004v2].

[2] T. Padmanabhan, "Equipartition of energy in the horizon degrees of freedom and the emergence of gravity," [arXiv gr-qc (Dec, 2009) 0912.3165v2].

[3] T. Padmanabhan, "Thermodynamical Aspects of Gravity: New insights," [arXiv gr-qc (Nov, 2009) 0911.5004v2].

[4] F. Caravelli and L. Modesto, "Holographic actions from black hole entropy," [arXiv gr-qc (Jan, 2010) 1001.4364v2].

[5] E. P. Verlinde, "On the Origin of Gravity and the Laws of Newton," [arXiv hep-th (Jan, 2010) 1001.0785v1].
[6] L. Smolin, "Newtonian gravity in loop quantum gravity," [arXiv gr-qc (Jan, 2010) 1001.3668v1].

[7] T. Padmanabhan, "Why Does the Universe Expand ?," [arXiv gr-qc (Jan, 2010) 1001.3380v1].

[8] R.-G. Cai, L.-M. Cao, and N. Ohta, "Friedmann Equations from Entropic Force," [arXiv hep-th (Jan, 2010) 1001.3470v1].

[9] M. Li and Y. Wang, "Quantum UV/IR Relations and Holographic Dark Energy from Entropic Force," [arXiv hep-th (Jan, 2010) 1001.4466v1].

[10] T. Wang, "The Coulomb Force as an Entropic Force," [arXiv hep-th (Jan, 2010) 1001.4965v1].

[11] Y. Zhang, Y. gui Gong, and Z.-H. Zhu, "Modified gravity emerging from thermodynamics and holographic principle," [arXiv hep-th (Jan, 2010) 1001.4677v1]. 
[12] Y. Ling, "A note on entropic force and brane cosmology," [arXiv hep-th (Jan, 2010) 1001.5324v1].

[13] S.-W. Wei, Y.-X. Liu, and Y.-Q. Wang, "Friedmann equation of FRW universe in deformed Horava-Lifshitz gravity from entropic force," [arXiv hep-th (Jan, 2010) $1001.5238 \mathrm{v} 1]$.

[14] Y. Wang, "Towards a Holographic Description of Inflation and Generation of Fluctuations from Thermodynamics," [arXiv hep-th (Jan, 2010) 1001.4786v2].

[15] J.-W. Lee, H.-C. Kim, and J. Lee, "Gravity from Quantum Information," [arXiv hep-th (Jan, 2010) $1001.5445 \mathrm{v} 1]$.

[16] T. Padmanabhan, "Entropy of Static Spacetimes and Microscopic Density of States," Class. Quant. Grav. 21 (2004) 4485-4494 [arXiv gr-qc (Jan, 2003) gr-qc/0308070v2].

[17] R.C. Tolman, "Relativity, Thermodynamics, And Cosmology", Oxford, 1934.

[18] J. Gibbs, "The collected works, Vol. 1, Thermodynamics", (Yale University Press, 1948).
[19] F. Weinhold, "Metric Geometry of equilibrium thermodynamics I, II, III, IV, V", J. Chem. Phys. 63, 2479, 2484, 2488, 2496 (1975); 65, 558 (1976).

[20] G. Ruppeiner, "Thermodynamics: A Riemannian geometric model", Phys. Rev. A 20, 1608 (1979).

[21] H. Quevedo, "Geometrothermodynamics," J.Math.Phys. 48 (2007) 013506 [arXiv physics.chem-ph (Apr, 2006) physics/0604164v2].

[22] H. Quevedo, "Geometrothermodynamics of black holes," Gen.Rel.Grav.40:971-984, 2008 [arXiv gr-qc (Apr, 2007) $0704.3102 v 2]$.

[23] P. Salamon, E. Ihrig and R.S. Berry, "A group of coordinate transformations which preserve the metric of Weinhold", J. Math. Phys. 24, 2515 (1983).

[24] T. Padmanabhan, "Surface density of spacetime degrees of freedom from equipartition law in theories of gravity," [arXiv gr-qc (Mar, 2010) 1003.5665v1]. 